\title{
A new symmetry of the spatially flat Einstein-Friedmann equations
}

\author{
Steve Dussault ${ }^{\mathrm{a}}$, Valerio Faraoni ${ }^{\mathrm{b}}$ \\ Department of Physics and Astronomy, Bishop's University, 2600 College Street, Sherbrooke, Québec J1M 1Z7, Canada
}

Received: 22 September 2020 / Accepted: 20 October 2020 / Published online: 30 October 2020

(C) The Author(s) 2020

\begin{abstract}
We report a new symmetry of the EinsteinFriedmann equations for spatially flat Friedmann- LemaîtreRobertson-Walker universes. We discuss its application to barotropic perfect fluids and its use as a solution-generating technique for scalar field universes.
\end{abstract}

\section{Introduction}

In general-relativistic cosmology, the matter content of the universe is typically modelled by a perfect fluid with energy density $\rho(t)$ and isotropic pressure $P(t)$ related by a barotropic equation of state $P=P(\rho)$. This fluid is described by the stress-energy tensor

$T_{a b}=(P+\rho) u_{a} u_{b}+P g_{a b}$,

where $u^{c}$ is the fluid four-velocity, which coincides with the time direction of comoving observers (here we follow the notations of Ref. [1], using units in which the speed of light is unity).

Assuming spatial homogeneity and isotropy, the geometry is necessarily the Friedmann-Lemaître-Robertson-Walker (FLRW) one, with line element

$$
\begin{aligned}
d s^{2}= & g_{a b} d x^{a} d x^{b}=-d t^{2} \\
& +a^{2}(t)\left(\frac{d r^{2}}{1-K r^{2}}+r^{2} d \Omega_{(2)}^{2}\right)
\end{aligned}
$$

in comoving coordinates $(t, r, \vartheta, \varphi)$, where $g_{a b}$ is the metric tensor, $a(t)$ is the cosmic scale factor, $K$ is the curvature index, and $d \Omega_{(2)}^{2}=d \vartheta^{2}+\sin ^{2} \vartheta d \varphi^{2}$ is the line element on the unit 2-sphere [1]. In this geometry, the Einstein field equations

$R_{a b}-\frac{1}{2} g_{a b} R+\Lambda g_{a b}=8 \pi G T_{a b}$

\footnotetext{
a e-mail: sdussault19@ubishops.ca

be-mail: vfaraoni@ubishops.ca (corresponding author)
}

(where $R_{a b}$ is the Ricci tensor, $R \equiv g^{a b} R_{a b}$, and $\Lambda$ is the cosmological constant) reduce to the Einstein-Friedmann equations of relativistic cosmology

$$
\begin{aligned}
& H^{2} \equiv\left(\frac{\dot{a}}{a}\right)^{2}=\frac{8 \pi G}{3} \rho+\frac{\Lambda}{3}-\frac{K}{a^{2}}, \\
& \dot{\rho}+3 H(P+\rho)=0, \\
& \frac{\ddot{a}}{a}=-\frac{4 \pi G}{3}(\rho+3 P)+\frac{\Lambda}{3},
\end{aligned}
$$

where an overdot denotes differentiation with respect to $t$, $H(t) \equiv \dot{a} / a$ is the Hubble function, and the constant $K$ describes a closed universe if it is positive, a spatially flat one if it vanishes, or a hyperbolic one if $K<0$.

Out of the three Einstein-Friedmann equations (7)-(9), only two are independent. If any two are given, the third one can be derived from them. Without loss of generality, we take the Friedmann equation (4) and the energy conservation equation (5) as primary, with the acceleration equation (6) following from them.

Symmetries are important for any physical theory and, naturally, there is a wealth of literature on the symmetries of the Einstein-Friedmann equations. Some of these studies are inspired by string dualities, although they are not always directly related to them [2-22]; others are based on methods of supersymmetric quantum mechanics [2325], and other times FLRW symmetries are studied in relation with solution-generating techniques (e.g., [2, 16, 18, 2642]). There is increasing interest also in symmetries of the Einstein-Friedmann equations formulated in conformal time in the context of Penrose's conformal cyclic cosmology [43] and of hidden conformal symmetries [44-46]. Further motivation comes from classic and recent studies of inflation in which the symmetries of the relevant equations are used to generate solutions from known ones with different scalar field potentials $[47,48]$. This goal can be achieved while preserving the slow-roll approximation while the old solution is mapped into a new one $[47,48]$. Here we propose a new 
symmetry of these equations and discuss its possible uses for FLRW universes filled with a perfect fluid with barotropic, linear, and constant equation of state and for scalar fields minimally coupled to the spacetime curvature. This symmetry generalizes one previously introduced in Ref. [2] (see also $[3,4,6,10,11,14,15,21])$ and studied recently in Ref. [26].

\section{A new symmetry of the Einstein-Friedmann equations}

The new symmetry transformation involves a rescaling of the comoving time $t$ (and, consequently, of the Hubble function $H$ and of all first time derivatives), and the fluid energy density and pressure. Since, in general, the pressure is rescaled differently than the energy density, the equation of state changes under the symmetry transformation. The EinsteinFriedmann equations remain invariant in form. This symmetry only applies to spatially flat universes and is given by

$$
\begin{aligned}
d t & \rightarrow d \bar{t}=f(\rho) d t \\
\rho & \rightarrow \bar{\rho}=\frac{\left(1-f^{2}\right)}{8 \pi G f^{2}} \Lambda+\frac{\rho}{f^{2}}=\frac{\left(1-f^{2}\right)}{f^{2}} \rho_{\Lambda}+\frac{\rho}{f^{2}}, \\
P & \rightarrow \bar{P}=-\bar{\rho}+\frac{\left[4 \pi G f-(8 \pi G \rho+\Lambda) f^{\prime}\right]}{4 \pi G f^{3}}(P+\rho) \\
& =-\bar{\rho}+\frac{\left[f-2\left(\rho+\rho_{\Lambda}\right) f^{\prime}\right]}{f^{3}}(P+\rho),
\end{aligned}
$$

where $f(\rho)$ is a regular, positive, and dimensionless function, $f^{\prime}$ is its derivative, and $\rho_{\Lambda} \equiv \Lambda /(8 \pi G)$ is the effective energy density of the cosmological constant. Since $\rho=\rho(t)$, the new differential $d \bar{t}=f(\rho(t)) d t$ is exact, with $\tilde{t}(t)=$ $\int d t f(\rho(t))$. The inverse transformation is

$$
\begin{aligned}
d t & =\frac{d \bar{t}}{f}, \\
\rho & =f^{2} \bar{\rho}+\left(f^{2}-1\right) \rho_{\Lambda}, \\
P+\rho & =\frac{f^{3}}{f-2\left(\rho+\rho_{\Lambda}\right) f^{\prime}}(\bar{P}+\bar{\rho}) .
\end{aligned}
$$

Using

$$
\begin{aligned}
\dot{a} & \equiv \frac{d a}{d t}=\frac{d a}{d \bar{t}} \frac{d \bar{t}}{d t}=f \frac{d a}{d \bar{t}}, \\
H & =f \bar{H} \equiv \frac{f}{a} \frac{d a}{d \bar{t}},
\end{aligned}
$$

and Eq. (11), the Friedmann equation (4) with $K=0$ yields

$f^{2} \bar{H}^{2}=\frac{8 \pi G}{3}\left(f^{2} \bar{\rho}+\frac{f^{2}}{8 \pi G} \Lambda\right)$

and, finally,

$\bar{H}^{2}=\frac{8 \pi G}{3} \bar{\rho}+\frac{\Lambda}{3}$.
Let us verify the covariant conservation equation in the barred variables. We have

$$
\begin{aligned}
\frac{d \bar{\rho}}{d \bar{t}} & =\frac{1}{f} \frac{d}{d \bar{t}}\left[\frac{\left(1-f^{2}\right)}{f^{2}} \rho_{\Lambda}+\frac{\rho}{f^{2}}\right] \\
& =\frac{f-2\left(\rho+\rho_{\Lambda}\right) f^{\prime}}{f^{4}} \dot{\rho},
\end{aligned}
$$

so that

$$
\begin{aligned}
\frac{d \bar{\rho}}{d \bar{t}} & +3 \bar{H}(\bar{P}+\bar{\rho}) \\
& =\left[\frac{f-2\left(\rho+\rho_{\Lambda}\right) f^{\prime}}{f^{4}}\right][\dot{\rho}+3 H(P+\rho)]=0
\end{aligned}
$$

by virtue of the "old" conservation equation (5). The acceleration equation in barred variables is automatically satisfied since it can be easily derived from the other two EinsteinFriedmann equations (16) and (18).

The symmetry (7)-(9) is not contained in those discussed in Refs. [22,49]. When $\Lambda=0$, Eq. (9) simplifies considerably and gives

$f(\rho)=\sqrt{\frac{\rho}{\bar{\rho}}}$,

and then the symmetry (7)-(9) reduces to the one found by Chimento [2] and studied recently in [26]. In fact, this transformation consists of [2]

$$
\begin{aligned}
\rho & \rightarrow \bar{\rho}(\rho), \\
H & \rightarrow \bar{H}=\sqrt{\frac{\bar{\rho}}{\rho}} H, \\
P+\rho & \rightarrow \bar{P}+\bar{\rho}=(P+\rho) \sqrt{\frac{\rho}{\bar{\rho}}} \frac{d \bar{\rho}}{d \rho} .
\end{aligned}
$$

The comparison of Eq. (20) and our Eq. (9) with $\Lambda=0$ yields $\bar{\rho}(\rho)=\rho / f^{2}$ and

$f(\rho)=\sqrt{\frac{\rho}{\bar{\rho}}}$,

using which, Eq. (22) gives our Eq. (9). Furthermore, using Eq. (13), the comoving time rescaling (7) gives immediately the rescaling (21) of the Hubble function. Therefore, our transformation (7)-(9) constitutes a generalization of the symmetry (20)-(22) of Ref. [2] to the case in which a cosmological constant $\Lambda$ is present.

A de Sitter universe fueled by a positive cosmological constant is a fixed point of the transformation. In fact, if $\Lambda>0$ and $P=\rho=0$, Eq. (9) yields $\bar{P}=-\bar{\rho}=$ const., while Eq. (9) gives

$\bar{\rho}=\frac{\left(1-f^{2}\right)}{f^{2}} \rho_{\Lambda} ;$

however, since $\bar{\rho}$ is constant, it must be $f=$ const. Then, the constant $f$ can be absorbed into a change of unit of the 
comoving time $t$ and set to unity. Hence, the transformation (7)-(9) reduces to the identity and maps a de Sitter space into itself.

Explicit examples of transformations mapping perfect fluid universes with an equation of state of the form $P=w \rho$, $w=$ const. into FLRW universes with a fluid with non-linear equations of state and $\Lambda$ are shown in the Appendix.

\section{Perfect fluids with $w=$ const.}

Perfect fluids with barotropic, linear, and constant equation of state of the form $P=w \rho$ with $w=$ const. are of great interest in cosmology. Therefore, it is particularly relevant to consider symmetry transformations that map one such fluid into another one. Beginning from a fluid with equation of state $P=w \rho$ and writing $\bar{P}=(\bar{w}+1) \bar{\rho}$, Eq. (9) gives

$\bar{w}+1=(w+1) \frac{\rho}{\rho+\left(1-f^{2}\right) \rho_{\Lambda}} \frac{f-2\left(\rho+\rho_{\Lambda}\right) f^{\prime}}{f} ;$

by imposing that $\bar{w} \equiv \bar{P} / \bar{\rho}=$ const. and introducing

$s \equiv \frac{\bar{w}+1}{w+1}=$ const.

one finds an ordinary differential equation that must be satisfied in order to take a perfect fluid with constant equation of state into another:

$f^{\prime}+\frac{(s-1) f}{2\left(\rho+\rho_{\Lambda}\right)}+s f\left(1-f^{2}\right) \frac{\rho_{\Lambda}}{2 \rho\left(\rho+\rho_{\Lambda}\right)}=0$.

This equation is nonlinear and, in general, it is difficult to find analytic solutions $f(\rho)$. However, we can present one. Consider the choice

$f(\rho)=\sqrt{\frac{\rho+\rho_{\Lambda}}{\gamma \rho^{\alpha}+\rho_{\Lambda}}}$,

where $\alpha$ and $\gamma$ are constants, with $\alpha$ dimensionless and $[\gamma]=$ $\left[\rho^{1-\alpha}\right]$.

Substituting the putative solution (28) into the first order equation (27), straightforward manipulations lead to

$(s-\alpha)\left(\rho+\rho_{\Lambda}\right)=0$,

which is satisfied if $\alpha=s$.

If $\rho=\rho_{\Lambda}$, then the perfect fluid is a cosmological constant, the solution is de Sitter space, $f=\sqrt{\frac{2}{\gamma \rho_{\Lambda}^{\alpha-1}+1}}$ is constant and can be absorbed into a redefinition of the unit of time.

Under the transformation (7)-(9) with the choice (28) of the function $f(\rho)$, the energy density and pressure transform as

$$
\rho \rightarrow \bar{\rho}=\gamma \rho^{\alpha},
$$

$P \rightarrow \bar{P}=\gamma[\alpha(w+1)-1] \rho^{\alpha}$.

Combining these two equations yields $\bar{P}=[\alpha(w+1)-1] \bar{\rho}$ or $\bar{P}=\bar{w} \bar{\rho}$ with

$\bar{w}=\alpha(w+1)-1$,

where the constant $\gamma$ disappears from the equation of state. Special choices of the constants $w$ and $\alpha$ include the following.

- $w=-1$ implies $\bar{w}=-1 \quad \forall \alpha$ : this is again the result that de Sitter space is a fixed point of the transformation.

- A dust with $w=0$ is mapped into a perfect fluid with $\bar{w}=\alpha-1$. If $\alpha=1$, a FLRW space with dust is a fixed point and is mapped into another FLRW space filled with dust. This is a special case of the more general result below.

- If $\alpha=1$, one has $\bar{w}=w$, that is, any fluid with $w=$ const. is a fixed point of the transformation with function

$$
f_{1}(\rho)=\sqrt{\frac{\rho+\rho_{\Lambda}}{\gamma \rho+\rho_{\Lambda}}} .
$$

This result could have been guessed from the fact that the (now dimensionless) constant $\gamma$ disappears from the barred equation of state, therefore one could choose it to be unity, making $f \equiv 1$.

- If $\alpha=4 / 3$, then $\bar{w}=(4 w+1) / 3$ and a dust $(w=0)$ is mapped into radiation $(\bar{w}=1 / 3)$.

- If $\alpha=3 / 2$, a radiation fluid ( $w=1 / 3)$ is mapped into a stiff fluid $(\bar{w}=1)$.

\section{Scalar fields}

A scalar field $\phi$ minimally coupled to the curvature in a FLRW space behaves as an irrotational perfect fluid [5056]. A choice of the scalar field potential $V(\phi)$ corresponds, roughly speaking, to a choice of the equation of state for the effective fluid, but this is not a one-to-one correspondence [51,57,58].

The energy density and pressure are

$\rho_{\phi}=\frac{\dot{\phi}^{2}}{2}+V(\phi)$,

$P_{\phi}=\frac{\dot{\phi}^{2}}{2}-V(\phi)$,

respectively. The covariant conservation equation for this effective fluid coincides with the Klein-Gordon equation

$\ddot{\phi}+3 H \dot{\phi}+\frac{d V}{d \phi}=0$. 
It is clear from Eqs. (34) and (35) that a free massless scalar field behaves like a stiff fluid with equation of state $P=$ $\rho$. We will attempt to generate scalar field solutions with a potential $V(\phi)$ in a spatially flat FLRW starting from this free field solution.

We begin by noting that, since $d \phi / d \bar{t}=f^{-1} d \phi / d t$, after the symmetry transformation (7)-(9), the "new" energy density of this effective scalar field fluid is

$$
\begin{aligned}
\bar{\rho} & =\frac{1}{2 f^{2}}\left(\frac{d \phi}{d t}\right)^{2}+\frac{\left(1-f^{2}\right)}{f^{2}} \rho_{\Lambda} \\
& =\frac{1}{2}\left(\frac{d \phi}{d \bar{t}}\right)^{2}+\frac{\left(1-f^{2}\right)}{f^{2}} \rho_{\Lambda} .
\end{aligned}
$$

By choosing

$f=\sqrt{\frac{\rho_{\Lambda}}{\rho_{\Lambda}+V(\phi)}}$,

where $V(\phi)$ is the scalar field potential that we want to obtain, one has

$\bar{\rho}=\frac{1}{2}\left(\frac{d \phi}{d \bar{t}}\right)^{2}+V(\phi)$,

which is the energy density of a new scalar field $\phi(\bar{t})$ obtained by changing the time variable $t \rightarrow \bar{t}(t)$. This transformation, which is only defined for a strictly positive $\Lambda$, is still implicit, as is the function $f(\rho)$ in Eq. (38), which can be written down explicitly only after expressing $\phi$ as a function of the "old" density $\rho$.

For a free scalar field $\phi$ in a FLRW universe, the KleinGordon equation (36) is immediately integrated to

$\dot{\phi}=\frac{C}{a^{3}}$,

where $C$ is an integration constant. If the energy density is written as $\rho=\rho_{0} / a^{6}$ as usual in the fluid description, then $\rho_{0}=C^{2} / 2$.

The scale factor for a stiff fluid plus positive cosmological constant is (see, e.g., [57,59])

$a(t)=a_{0}[\sinh (\sqrt{3 \Lambda} t)]^{1 / 3}$,

where

$a_{0}=\left(\frac{\rho_{0}}{\rho_{\Lambda}}\right)^{1 / 6}=\left(\frac{C^{2}}{2 \rho_{\Lambda}}\right)^{1 / 6}$.

We can then integrate explicitly the first integral (40) obtaining

$\phi(t)=\frac{C}{a_{0}^{3}} \int \frac{d t}{\sinh (\sqrt{3 \Lambda} t)}=\phi_{0} \ln \left[\tanh \left(\frac{\sqrt{3 \Lambda}}{2} t\right)\right]$, where

$\phi_{0}=\frac{C}{\sqrt{3 \Lambda} a_{0}^{3}}$.

The energy density is

$\rho(t)=\frac{\dot{\phi}^{2}}{2}=\frac{C^{2}}{2 a_{0}^{6}} \frac{1}{\sinh ^{2}(\sqrt{3 \Lambda} t)}$.

Let us express $\rho$ as a function of the scalar field $\phi$; if this relation can be inverted, we will then obtain the explicit form of the function $f(\rho)$ that achieves Eq. (39).

Exponentiating both sides of Eq. (43) gives

$\mathrm{e}^{\phi / \phi_{0}}=\tanh \left(\frac{\sqrt{3 \Lambda} t}{2}\right)$

and algebraic manipulations yield

$\mathrm{e}^{\sqrt{3 \Lambda} t}=-\operatorname{coth}\left(\frac{\phi}{2 \phi_{0}}\right)$

Then, it is straightforward to obtain

$\sinh (\sqrt{3 \Lambda} t)=-\frac{1}{\sinh \left(\phi / \phi_{0}\right)}$

and finally Eq. (45) gives

$\phi=\phi_{0} \operatorname{arcsinh}\left(\frac{\sqrt{2} a_{0}^{3}}{C} \sqrt{\rho}\right)$,

which allows us to write explicitly the function $f(\rho)$ appearing in the symmetry transformation as

$$
\begin{aligned}
f(\rho) & =\frac{1}{\sqrt{1+V / \rho_{\Lambda}}} \\
& =\frac{1}{\sqrt{1+V\left(\phi_{0} \operatorname{arcsinh}\left(\sqrt{2} a_{0}^{3} \sqrt{\rho} / C\right)\right) / \rho_{\Lambda}}} .
\end{aligned}
$$

In terms of the comoving time $t$, we have

$$
f(t)=\frac{1}{\sqrt{1+V\left(\phi_{0} \ln \tanh \left(\frac{\sqrt{3 \Lambda} t}{2}\right)\right) / \rho_{\Lambda}}} .
$$

The solution is not complete, though, because we need $\phi(\bar{t})$ instead of $\phi(t)$, which means using the first transformation equation (7) to obtain $\bar{t}(t)$ and then inverting this relation and substituting the result into Eq. (43). This means computing explicitly the integral

$\bar{t}(t)=\int \frac{d t}{\sqrt{1+V\left(\phi_{0} \ln \tanh \left(\frac{\sqrt{3 \Lambda} t}{2}\right)\right) / \rho_{\Lambda}}}$,

and then inverting this relation to obtain $t(\bar{t})$. Unfortunately, these steps are usually not possible. 
Table 1 The possible combinations of variables involved in a symmetry transformation of the Einstein-Friedmann equations

\begin{tabular}{llll}
\hline$\rho, a, t, P$ & $\rho, a, t$ & $\rho, a, P$ & $\rho, t, P$ \\
$a, t, P$ & $H, \rho, P$ & $\rho, a$ & $\rho, t$ \\
$\rho, P$ & $a, t$ & $a, P$ & $t, P$ \\
$H, \rho$ & $H, P$ & - & - \\
\hline
\end{tabular}

\section{Conclusions}

General symmetries of the Einstein-Friedmann equations involve transformations of the variables $a, t, H, \rho$, and $P$. If $a$ or $t$ are transformed, $H$ cannot be transformed, and if $H$ is transformed (as in [2]), then the only remaining choices are transforming $\rho$ or $P$, since $H$ is constructed out of $a$ and $t$.

Here, the term "general" symmetry means that no relation is imposed between any two variables. For example, it is common in cosmology to impose the equation of state $P=w \rho$ with $w=$ const. while searching for symmetries (as in [49]), in which case one transforms the set of variables $(\rho, a, t, P)$, but the relation imposed between $P$ and $\rho$ restrict the generality. Table 1 reports the possible combinations of variables involved in a symmetry transformation of the Einstein-Friedmann equations.

The $(H, \rho, P)$ case has already been partially covered by Chimento [2]. In this work, we have studied an extension of his symmetry transformations for $\Lambda>0$. Any symmetry transforming the scale factor cannot be made more general, because it demands a restriction of variables (these symmetries are still of interest when the barotropic, linear, and constant equation of state $P=w \rho$ is imposed).

The possible general symmetries transforming only two variables include $(\rho, t),(\rho, P),(t, P),(H, \rho)$, and $(H, P)$. However, it can be easily shown that these are not suitable for generating general symmetries. Only one possible general symmetry remains, i.e., the one involving $(\rho, t, P)$ and, to the best of our knowledge, this was not discussed in the literature yet (however, if one does not touch $a$, rescaling the time $t$ is equivalent to rescaling $H$ together with $\rho$ and $P$, which was done in [2] for vanishing cosmological constant).

The situation in which the new symmetry reported here would be most useful is in the generation of new scalar field solutions for a specified potential $V(\phi)$ from the corresponding solution for a free scalar field (plus positive $\Lambda$ ). Unfortunately, although the generating method exposed in Sect. 4 carries through almost to the end, two obstacles will, in general, forbid one to obtain results. First, one needs to compute explicitly the integral (52) in terms of elementary functions, which in general is not possible. Second, one needs to invert analytically the relation $\bar{t}(t)$ thus obtained, which is also, by all means, not guaranteed. It is interesting, however, that a tall order such as generating solutions of the non-linear EinsteinFriedmann-Klein-Gordon equations from a free field solution can be carried through to such an extent. In the case $\Lambda=0$ studied in [2], one cannot generate a potential $V(\phi)$ as we did here and the free scalar field remains free after the transformation is performed.

Overall, the conclusion emerging is that the general transformations of the kind (7)-(9) (or their restriction to $\Lambda=0$ studied in [2]) are not very useful in generating new inflationary solutions for the early universe as one would have hoped.

Acknowledgements This work is supported, in part, the Natural Sciences \& Engineering Research Council of Canada (Grant No. 201603803 to V.F.) and by Bishop's University.

Data Availability Statement This manuscript has no associated data or the data will not be deposited. [Authors' comment: There are no data in this article because its nature is theoretical and mathematical.]

Open Access This article is licensed under a Creative Commons Attribution 4.0 International License, which permits use, sharing, adaptation, distribution and reproduction in any medium or format, as long as you give appropriate credit to the original author(s) and the source, provide a link to the Creative Commons licence, and indicate if changes were made. The images or other third party material in this article are included in the article's Creative Commons licence, unless indicated otherwise in a credit line to the material. If material is not included in the article's Creative Commons licence and your intended use is not permitted by statutory regulation or exceeds the permitted use, you will need to obtain permission directly from the copyright holder. To view a copy of this licence, visit http://creativecomm ons.org/licenses/by/4.0/.

Funded by $\mathrm{SCOAP}^{3}$.

\section{Appendix A: Explicit examples of symmetry transforma- tions}

Here we show other explicit examples of transformations of the kind (7)-(9) that carry a FLRW universe with a perfect fluid and $\Lambda>0$ into one with a fluid with non-linear equations of state plus $\Lambda$.

For perfect fluids with constant equation of state $P=w \rho$, the transformed equation of state is

$\bar{P}=-\bar{\rho}+(w+1) \frac{\left[f-2\left(\rho+\rho_{\Lambda}\right) f^{\prime}\right]}{f^{3}} \rho ;$

this expression neds to be rewritten in terms of barred variables only. To this end, one can search for a function $f(\rho)$ such that

$$
\begin{array}{r}
\frac{\left[f-2\left(\rho+\rho_{\Lambda}\right) f^{\prime}\right]}{f^{3}} \rho=g(\bar{\rho}) \\
=g\left(\frac{\left(1-f^{2}\right)}{f^{2}} \rho_{\Lambda}+\frac{\rho}{f^{2}}\right) .
\end{array}
$$


Certain functions $g(\bar{\rho})$ allow the above ordinary differential equation to be solved for interesting equations of state of the form

$\bar{P}=\left[\frac{g(\bar{\rho})}{\bar{\rho}}(w+1)-1\right] \bar{\rho}$.

Solving Eq. (A.2) with $g(\bar{\rho})=\alpha \bar{\rho}$ we obtain

$f(\rho)=\sqrt{\frac{\left(\rho+\rho_{\Lambda}\right)}{\gamma \rho^{\alpha}+\rho_{\Lambda}}}$,

which brings a perfect fluid with constant equation of state into another one, and has been studied in Sect. 3 .

Another example is given by solving Eq. (A.2) with $g(\bar{\rho})=\alpha \tanh \bar{\rho}$, which gives

$f(\rho)=\sqrt{\frac{\rho+\rho_{\Lambda}}{\operatorname{arcsinh}\left(\gamma \rho^{\alpha}\right)+\rho_{\Lambda}}}$,

where $\alpha$ and $\gamma$ are constants, with $\alpha$ dimensionless and $[\gamma]=$ $\left[\rho^{-\alpha}\right]$. Then, one obtains

$\bar{\rho}=\operatorname{arcsinh}\left(\gamma \rho^{\alpha}\right)$

and

$\bar{P}=\alpha(w+1) \tanh \bar{\rho}-\bar{\rho}$.

The third example is generated by solving Eq. (A.2) with $g(\bar{\rho})=\alpha \mathrm{e}^{\beta(\bar{\rho})}$. This solution generates

$f(\rho)=\sqrt{-\frac{\beta\left(\rho+\rho_{\Lambda}\right)}{\ln \left[\alpha \beta\left(\gamma-\ln \left(\rho / \rho_{0}\right)\right)\right]-\beta \rho_{\Lambda}}}$,

with $\alpha, \beta$, and $\gamma$ dimensionless constants, while $\rho_{0}$ is a constant with the dimensions of an energy density. This choice gives

$\bar{\rho}=-\frac{\ln \left\{\alpha \beta\left[\gamma-\ln \left(\rho / \rho_{0}\right)\right]\right\}}{\beta}$

and

$\bar{P}=\alpha(w+1) \mathrm{e}^{\beta \bar{\rho}}-\bar{\rho}$.

A fourth example is given by the solution $g(\bar{\rho})=\alpha / \bar{\rho}$ of Eq. (A.2), which yields

$f(\rho)=\sqrt{\frac{\rho+\rho_{\Lambda}}{\sqrt{\gamma+2 \alpha \ln \left(\rho / \rho_{0}\right)}+\rho_{\Lambda}}}$,

and

$$
\begin{aligned}
& \bar{\rho}=\sqrt{\gamma+2 \alpha \ln \left(\rho / \rho_{0}\right)}, \\
& \bar{P}=\frac{\alpha(w+1)}{\bar{\rho}}-\bar{\rho} .
\end{aligned}
$$

Our last example is generated by $g(\bar{\rho})=\alpha \bar{\rho}^{2}$ and the subsequent

$$
\begin{aligned}
f(\rho)= & {\left[\alpha\left(\ln \left(\rho / \rho_{0}\right)+\gamma\right)\left(\alpha \rho_{\Lambda} \ln \left(\rho / \rho_{0}\right)+\alpha \gamma \rho_{\Lambda}+1\right)\right]^{1 / 2} } \\
& \cdot \frac{\sqrt{\rho+\rho_{\Lambda}}}{\alpha\left[\ln \left(\rho / \rho_{0}\right)+\gamma\right] \rho_{\Lambda}},
\end{aligned}
$$

which gives

$\bar{\rho}=-\frac{\rho_{\Lambda}}{\alpha\left(\ln \left(\rho / \rho_{0}\right)+\gamma\right) \rho_{\Lambda}+1}$

and

$\bar{P}=[\alpha \bar{\rho}(w+1)-1] \bar{\rho}$.

Rather cumbersome symmetry mappings, that we do not report, are generated by the solutions $g(\bar{\rho})=\alpha \bar{\rho}^{3}, \alpha \bar{\rho}^{4}$ of Eq. (A.2). For powers of $\bar{\rho}$ higher than four, the relevant expressions become very long and involved.

\section{References}

1. R.M. Wald, General Relativity (Chicago University Press, Chicago, 1984)

2. L.P. Chimento, Symmetry and inflation. Phys. Rev. D 65, 063517 (2002)

3. J.M. Aguirregabiria, L.P. Chimento, A. Jakubi, R. Lazkoz, Symmetries leading to inflation. Phys. Rev. D 67, 083518 (2003)

4. L.P. Chimento, R. Lazkoz, On the link between phantom and standard cosmologies. Phys. Rev. Lett. 91, 211301 (2003)

5. M.P. Dabrowski, T. Stachowiak, M. Szydlowski, Phantom cosmologies. Phys. Rev. D 68, 103519 (2003)

6. J.M. Aguirragabiria, L.P. Chimento, R. Lazkoz, Phantom k-essence cosmologies. Phys. Rev. D 70, 023509 (2004)

7. G. Calcagni, Patch dualities and remarks on nonstandard cosmologies. Phys. Rev. D 71, 023511 (2005)

8. M. Szydlowski, W. Godlowski, R. Wojtak, Equation of state for Universe from similarity symmetries. Gen. Relativ. Gravit. 38, 795 (2006)

9. L.P. Chimento, R. Lazkoz, Duality extended Chaplygin cosmologies with a big rip. Class. Quantum Gravity 23, 3195-3204 (2006)

10. L.P. Chimento, W. Zimdhal, Duality invariance and cosmological dynamics. Int. J. Mod. Phys. D 17, 2229-2254 (2008)

11. L.P. Chimento, D. Pavon, Dual interacting cosmologies and late accelerated expansion. Phys. Rev. D 73, 063511 (2006). https:// doi.org/10.1103/PhysRevD.73.063511

12. M.P. Dabrowski, C. Kiefer, B. Sandhoefer, Quantum phantom cosmology. Phys. Rev. D 74, 044022 (2006)

13. Y.-F. Cai, H. Li, Y.-S. Piao, X. Zhang, Duality invariance and cosmological dynamics. Phys. Lett. B 646, 141 (2007)

14. L.P. Chimento, F.P. Devecchi, M.I. Forte, G.M. Kremer, Phantom cosmologies and fermions. Class. Quantum Gravity 25, 085007 (2008)

15. M. Cataldo, L.P. Chimento, Form invariant transformations between n-dimensional flat Friedmann-Robertson-Walker cosmologies. Int. J. Mod. Phys. D 17, 1981-1989 (2008)

16. S. Capozziello, E. Piedipalumbo, C. Rubano, P. Scudellaro, Noether symmetry approach in phantom quintessence cosmology. Phys. Rev. D 80, 104030 (2009)

17. J. Wang, T. Lan, S.-P. Yang, Cosmic duality and statefinder diagnosis of spinor quintom. J. Theor. Phys. 1, 62 (2012) 
18. S. Capozziello, V. Faraoni, Beyond Einstein Gravity (Springer, New York, 2010)

19. Y.-F. Cai, E.N. Saridakis, M.R. Setare, J.-Q. Xia, Quintom cosmology: Theoretical implications and observations. Phys. Rep. 493, 1-60 (2010)

20. L. Pucheu, M. Bellini, Phantom and inflation scenarios from a 5D vacuum through form-invariance transformations of the Einstein equations. Nuovo Cimento B 125, 851-859 (2010)

21. L.P. Chimento, R. Lazkoz, M.G. Richarte, Inflation in the DiracBorn-Infeld framework. Phys. Rev. D 83, 063505 (2011)

22. V. Faraoni, A symmetry of the spatially flat Friedmann equations with barotropic fluids. Phys. Lett. B 703, 228-231 (2011)

23. H.C. Rosu, K.V. Khmelnytskaya, Shifted Riccati procedure: Application to conformal barotropic FRW cosmologies. SIGMA 7, 013 (2011)

24. H.C. Rosu, P. Ojeda-May, Supersymmetry of FRW barotropic cosmologies. Int. J. Theor. Phys. 45, 1191 (2006)

25. M. Nowakoswki, H.C. Rosu, Newtons' Laws of motion in the form of a Riccati equation. Phys. Rev. E 65, 047602 (2002). https://doi. org/10.1103/PhysRevE.65.047602

26. T. Pailas, N. Dimakis, A. Paliathanasis, P.A. Terzis, T. Christodoulakis, Infinite dimensional symmetry groups of the Friedmann equations. arXiv:2005.11726 [gr-qc]

27. T. Christodoulakis, A. Karagiorgos, A. Zampeli, Symmetries in classical and quantum treatment of Einstein's cosmological equations and mini-superspace actions. Symmetry 10, 70 (2018)

28. M. Tsamparlis, A. Paliathanasis, Symmetries of differential equations in cosmology. Symmetry 10, 233 (2018)

29. G. Gionti, A. Paliathanasis, Duality transformation and conformal equivalent scalar-tensor theories. Mod. Phys. Lett. A 33, 1850093 (2018)

30. A. Paliathanasis, M. Tsamparlis, S. Basilakos, J.D. Barrow, Barrow, Classical and quantum solutions in Brans-Dicke cosmology with a perfect fluid. Phys. Rev. D 93, 043528 (2016)

31. A. Paliathanasis, S. Capozziello, Noether symmetries and duality transformations in cosmology. Mod. Phys. Lett. A 31, 1650183 (2016)

32. A. Zampeli, T. Pailas, P.A. Terzis, T. Christodoulakis, Conditional symmetries in axisymmetric quantum cosmologies with scalar fields and the fate of the classical singularities. J. Cosmol. Astropart. Phys 05, 066 (2016)

33. T. Christodoulakis, N. Dimakis, P.A. Terzis, B. Vakili, E. Melas, T. Grammenos Minisuperspace canonical quantization of the Reissner-Nordström black hole via conditional symmetries. Grammenos, Phys. Rev. D 89, 044031 (2014)

34. A. Paliathanasis, M. Tsamparlis, Two scalar field cosmology: Conservation laws and exact solution. Phys. Rev. D 90, 043529 (2014)

35. T. Christodoulakis, N. Dimakis, P.A. Terzis, Lie point and variational symmetries in minisuperspace Einstein gravity. J. Phys. A Math. Theor. 47, 095202 (2014)

36. N. Dimakis, T. Christodoulakis, P.A. Terzis, FLRW metric cosmology with a perfect fluid by generating integrals of motion. J. Geom. Phys. 77, 97 (2012)

37. S. Basilakos, M. Tsamparlis, A. Paliathanasis, Using the Noether symmetry approach to probe the nature of dark energy. Phys. Rev. D 83, $103512(2011)$

38. A. Paliathanasis, M. Tsamparlis, S. Basilakos, Constraints and analytical solutions of $f(R)$ theories of gravity usingNoether symmetries. Phys. Rev. D 84, 123514 (2011)

39. A. Borowiec, S. Capozziello, M. De Laurentis, F.S.N. Lobo, A. Paliathanasis, M. Paolella, A. Wojnar, Invariant solutions and Noether symmetries in hybrid gravity. Phys. Rev. D 91, 023517 (2015)

40. S. Capozziello, M. De Laurentis, R. Myrzakulov, Noether symmetry approach for teleparallel-curvature cosmology. Int. J. Geom. Meth. Mod. Phys. 12, 1550095 (2015)
41. S. Capozziello, M. De Laurentis, K.F. Dialektopoulos, Dialektopoulos, Noether symmetries in Gauss-Bonnet-teleparallel cosmology. Eur. Phys. J. C 76, 629 (2016)

42. E. Piedipalumbo, M. De Laurentis, S. Capozziello, Noether symmetries in interacting quintessence cosmology. Phys. Dark Univ. 27, 100444 (2020)

43. J. Alberto Vazquez, S. Hee, M.P. Hobson, A.N. Lasenby, M. Ibison, M. Bridges, Observational constraints on conformal time symmetry, missing matter and double dark energy. JCAP 07, 062 (2018)

44. J. Ben Achour, E.R. Livine, J. High Energy Phys. 12, 031 (2019). arXiv: 1909.13390 [gr-qc]

45. J. Ben Achour, E.R. Livine, The cosmological constant from conformal transformations: Möbius invariance and Schwarzian action. arXiv:2004.05841 [gr-qc]

46. J. Ben Achour, E.R. Livine, Conformal structure of FLRW cosmology: Spinorial representation and the so $(3,2)$ algebra of observables. J. High Energy Phys. 03, 067 (2020). arXiv:2001.11807 [grqc]

47. P. Parsons, J.D. Barrow, New inflation from old. Class. Quant. Gravit. 12, 1715 (1995)

48. J.D. Barrow, A. Paliathanasis, Reconstructions of the dark-energy equation of state and the inflationary potential. Gen. Relativ. Gravit. 50, 82 (2018). https://doi.org/10.1007/s10714-018-2402-4

49. V. Faraoni, A symmetry of the Einstein-Friedmann equations for spatially flat, perfect fluid, universes. Symmetry 12, 147 (2020). https://doi.org/10.3390/sym12010147

50. M.S. Madsen, Scalar fields in curved spacetimes. Class. Quant. Gravit. 5, 627 (1988)

51. M.S. Madsen, A note on the equation of state of a scalar field. Astrophys. Space Sci. 113, 205 (1985)

52. L.O. Pimentel, Energy-momentum tensor in the general scalartensor theory. Class. Quant. Gravit. 6, L263 (1989)

53. V. Faraoni, Correspondence between a scalar field and an effective perfect fluid. Phys. Rev. D 85, 024040 (2012)

54. I. Semiz, Comment on "Correspondence between a scalar field and an effective perfect fluid". Phys. Rev. D 85, 068501 (2012)

55. G. Ballesteros, D. Comelli, L. Pilo, Thermodynamics of perfect fluids from scalar field theory. Phys. Rev. D 94, 025034 (2016)

56. V. Faraoni, J. Côté, Imperfect fluid description of modified gravitie. Phys. Rev. D 98, 084019 (2018)

57. V. Faraoni, A new solution for inflation. Am. J. Phys. 69, 372 (2001)

58. S.Ş. Bayin, F.I. Cooperstock, V. Faraoni, A singularity-free cosmological model with a conformally coupled scalar field. Astrophys. J. 428, 439 (1994)

59. P.H. Chavanis, Cosmology with a stiff matter era. Phys. Rev. D 92 , 103004 (2015) 